\title{
REVIEW
}

\section{'Cooperative sedation': optimizing comfort while maximizing systemic and neurological function}

\author{
Haley Goodwin, John J Lewin and Marek A Mirski* \\ This article is one of eleven reviews selected from the Annual Update in Intensive Care and Emergency Medicine 2012 (Springer Verlag, \\ DOI: 10.1007/978-3-642-25716-2) and co-published as a series in Critical Care. Other articles in the series can be found online at \\ http://ccforum.com/series/annualupdate2012. Further information about the Annual Update in Intensive Care and Emergency Medicine is \\ available from http://www.springer.com/series/8901.
}

\begin{abstract}
Introduction
Common indications for sedation in the intensive care unit (ICU) include patient comfort, management of agitation, pain, ventilator dysynchrony, and intracranial hypertension. Current trends in ICU care, however, have shifted towards sedation strategies that provide the minimally effective amount of sedation, to improve patient autonomy and preserve both the neurological exam and neurocognitive function.
\end{abstract}

\section{Long term cognitive effects of critical illness}

Perhaps the earliest investigations to realize severe cognitive deficits following ICU care were studies that evaluated patients post-coronary artery bypass graft (CABG) surgery [1]. The investigators of one study found that $53 \%$ of such patients demonstrated cognitive dysfunction at hospital discharge, and $42 \%$ exhibited continued cognitive dysfunction at the five-year follow-up visit [2]. Patients with acute respiratory distress syndrome (ARDS) have also received significant attention as relates long-term outcomes following ICU discharge and, notably, such patients have not had a surgical intervention that may have independently resulted in neurological sequelae. One study reported that $100 \%$ of ARDS patients had cognitive impairments at hospital discharge and, at the one-year follow-up visit, $78 \%$ had at least one persistent impairment relating to cognition [3]. Other reported data support that patients with general medical problems often experience cognitive impairments following ICU discharge [1]. Although clinical review suggests that the mechanism of cerebral dysfunction following critical illness may most often be related to hypoxia and hypoperfusion, other factors, such as metabolic encephalopathy, delirium, and embolic phenomena, have also been

*Correspondence: mmirski1@jhmi.edu

Johns Hopkins Medical Institutions, Baltimore, MD, USA implicated [1,3]. Regardless of the etiology of neurocognitive deficits, strategies that target a lighter, 'cooperative' depth of sedation would allow the primary team to identify changes in mental status and cognition in the early phase of critical illness when the condition may still be reversible.

\section{Daily awakenings}

The initial therapeutic intervention that evaluated the benefit of reducing the depth or interval of sedation was in the context of introducing daily prescribed awakenings or brief 'off-periods' for sedative medications. The hypothesis entertained was that such interval off-periods, even if for only a brief period, would enhance the ability of the ICU team to assess and accelerate ventilator weaning, and thus may lead to a reduction in mean duration of mechanical ventilation and a shorter ICU length of stay (LOS). Hence, the primary outcome measure has typically been time to extubation as compared to a controlled ICU ventilated population.

The first trial evaluating the daily interruption of sedation was performed as a single center study by Kress and colleagues [4]. The investigators of this important study randomized patients to either a daily interruption of sedation $(n=68)$ or standard practice $(n=60)$. In both groups, sedative agents were titrated by nurses based on standard protocols to achieve a Ramsey Score of 3-4 (responsive to commands or wake briskly to the sound of a loud noise). In the intervention group, sedation was stopped daily, beginning on the $3^{\text {rd }}$ ventilated day, until the patient could perform at least three of the following activities: Open eyes in response to voice, track the investigator with eyes, squeeze hand or stick out tongue upon request. Kress and colleagues found that this practice of daily sedation interruption led to fewer days requiring mechanical ventilation and shorter LOS in the 
ICU. In addition, as a secondary outcome measure, patients in the intervention group required fewer imaging studies to investigate poor neurologic function. Despite the positive findings, the study was too small and not sufficiently powered to rigorously assess the effect on patient safety. In addition, patients in this study were managed solely in the medical ICU, so the role of daily interruption of sedation in other ICU populations remained unclear [4].

A second study was published in 2008 that coupled spontaneous awakening trials to a spontaneous breathing trial (SBT) [5]. All patients in the trial were screened for SBT, but only the intervention group paired the latter with the spontaneous awakening trials. This trial also demonstrated the positive effects of daily interruption of sedation, which included increased ventilator-free days and reduced ICU and hospital LOS. Although there were more self-extubations in the intervention group compared to the control group, the rate of re-intubation was similar between groups. In addition, this study demonstrated a statistically significant $32 \%$ reduction in deaths at one year [5].

In 2010, a group of investigators took the concept of daily awakenings one step further, in fact challenging the need to routinely offer continuous sedation [6]. One hundred and twenty-eight patients were randomized to receive either sedation with a combination of propofol and midazolam or no sedation at all. In the sedation group, patients participated in daily awakening trials similar to those described in a previous study [4]. The group receiving no sedation required significantly fewer days of mechanical ventilation, in addition to shorter ICU and hospital LOS [6].

One concern for limiting sedation is the psychological impact it may have on the survivors of intensive care. The development of post-traumatic stress disorder (PTSD) has been reported to varying degrees after discharge from an ICU [7]. In order to evaluate the effect of daily awakenings on the development of PTSD, patients were evaluated for the presence of PTSD by a clinical psychologist [8]. Some of the patients included were in the trial evaluating the daily interruption of sedation mentioned earlier in this chapter [4]. Alhough chronic anxiety and mild depression were found in both the control and daily interruption groups, PTSD was identified in 6 of 19 patients in the control group. None of the 13 patients in the daily interruption group developed PTSD. Interestingly, no patient in the daily interruption group even recalled any of the awakened periods [8]. These data suggest that daily interruption of sedation does not have a detrimental effect on the long-term psychological outcome of patients and may even have a protective effect, though that could not be determined solely based on this report.

\section{Early mobilization}

In addition to the long-term neurocognitive effects, it is well known that critical illness can cause a variety of neuromuscular sequelae, including muscle wasting from inactivity, critical illness myopathy and polyneuropathy [9]. These disorders contribute to prolonged functional disability after ICU discharge [9]. In one study of 87 survivors of ARDS who were evaluated at the one-year mark [10], only $49 \%$ had returned to work, and patients reported muscle weakness and chronic fatigue as reasons for unemployment. In addition, at one year, patients were only able to walk $66 \%$ of the predicted distance during a 6-minute walk test [10], further supporting the long lasting impact of critical illness.

Prolonged inactivity is thought to be a contributor to neuromuscular illness in the intensive care unit. In order to refute the concept that immobility is required for mechanically ventilated patients, a group in Salt Lake City conducted a feasibility trial for early mobility in 103 ventilated patients [11]. Early mobility was measured by the ability of the patient to sit on the edge of the bed, sit in a chair, and ambulate with or without assistance. The investigators demonstrated that $69.4 \%$ of patients were able to walk a minimum of 200 feet, with less than $3 \%$ being incapable of activity during their ICU admission. This impressive level of mobilization was achieved at the expense of very few adverse events; a total of 14 occurred over 1,449 activity events, including falls to the knees without injury, acute desaturation, changes in systolic blood pressure, and removal of feeding tube [11].

After the feasibility study had been conducted, other studies followed providing further evidence in support of early patient mobilization prior to liberation from mechanical ventilators [12-14]. One randomized controlled trial demonstrated that early mobilization was associated with more frequent return to independent functional status at hospital discharge and more frequent discharge home [14]. In addition, mobility was associated with a reduction in the duration of delirium during hospitalization.

In each of the above studies, the intervention involved a multidisciplinary team. Members included respiratory, physical, and occupational therapists, a nurse and a critical care technician [11-14]. In addition to the team, it was concluded that a coordinated sedation strategy was important to the success of the early mobilization intervention. One of the clinical studies specifically combined the intervention of early mobilization with daily interruption of sedation [14], although clearly such effort placed to coordinate such activities may not be readily translated to usual ICU practice. In all these trials, it was noted that lower depth of sedation was a significant predictor for success. Hence, minimizing the use of sedation, or eliminating it completely may increase the 
ability to successfully involve an interdisciplinary team in mobilizing mechanically ventilated patients.

\section{Neurological monitoring}

As more patients are admitted to the ICU and survive the experience, more attention is being given to the neurologic complications of critical illness, especially persistent neurocognitive dysfunction as it may occur even following admission for a non-neurological condition. Despite the brain being widely recognized as the most important organ, it often receives little attention during critical care management. This is likely due to several factors. One, the primary admission diagnosis to the ICU is nonneurologic, thus the primary focus lies initially elsewhere. Second, often the true neurologic status of the patient is unclear, as it may be masked by desirable or necessary sedation, alone or together with the addition of pharmacological paralysis, rendering neurological evaluation difficult or impossible.

Fortunately, the common-held belief in ICU practice that patient sedation de facto leads to an un-examinable patient is now fully in jeopardy. Previously cited studies have confirmed both the capability and safety of reducing the depth of sedation for the benefits of systemic physiology. There is also evidence that sedation need not be incompatible with preservation of intellectual capacity. Mirski and colleagues demonstrated in the Acute Neurological ICU Sedation Trial (ANIST) that anxious or agitated ventilator-dependent patients tolerated well a light plane of continuous sedation - 'cooperative sedation' - and were able to participate in neurocognitive evaluations [15]. Important to this fundamental acknowledgement, it was observed that neurocognitive capacity could be maintained or even improved during active continuous sedation as the disruptive influences of anxiety and agitation on concentration were eliminated. Dexmedetomidine, specifically, was shown not to reduce intellectual capacity during periods of cooperative sedation in contrast to low-dose infusion of propofol [15]. Other sedatives may also be effective in low doses.

The use of minimal to no sedation may have many benefits that have yet to be discovered. In addition to reduction in days requiring mechanical ventilation, shorter durations of stay, fewer diagnostic scans, and less PTSD, awake and cooperative patients should be able to be more engaged in their own care and decision making, and play a more significant role in their own recovery.

\section{Monitoring sedation}

Sedation scales play an enormous role in the evaluation and communication of sedating therapies in the ICU. For patients requiring sedation, there are many validated scales available to assess the levels of consciousness and arousal. Perhaps the most widely used scale is the
Richmond Agitation-Sedation Scale (RASS) [16,17]. Other scales available include the Riker Sedation-Agitation Scale (SAS) [18], the Motor Activity Assessment Scale [19], the Adaptation to the Intensive Care Environment (ATICE) [20], and the Nursing Instrument for the Communication of Sedation (NICS) [21]. Although each scale is unique, the over-all objective of the sedation scales is to allow for the assessment of goal-directed sedation for each patient. Although phrased differently, each scale has a value that represents calm and alert. Whereas the optimum level of sedation may be 'awake and cooperative', needs may vary greatly among ICU patients. Sedatives are often administered for therapeutic reasons other than sedation. Examples include reduction of cerebral metabolic rate of oxygen consumption and subsequent lowering of intracranial pressure (ICP), ventilator dysynchrony, or withdrawal syndromes. Thus, it is imperative to use a scale that can represent many levels of sedation, as targets may differ depending on the therapeutic intent.

Other objective tools have been studied for the assessment of sedation in critically ill patients. One that has perhaps received the most attention is the bispectral index, a cerebral function monitor that uses data based on the raw electroencephalograph (EEG) to quantify a patient's level of arousal. The bispectral index allows for continuous monitoring of sedation with the benefit of assigning an ordinal score to relative EEG wave form activity that lacks the subjectivity of the sedation scales. This technology has been used to assess depth of anesthesia in the operating room and has been shown, in that arena, to have reduced the number of episodes of intraoperative awareness [22]. The bispectral index has also been studied in the ICU in several populations of patients including medical, brain-injured and cardiac surgery patients and has been tested against several different sedation scales, including the visual analog scales, the Ramsey scale, RASS and SAS [23-26]. Although the bispectral index correlates to varying degrees with validated sedation scales, the wide-spread use of bispectral index for ICU sedation has not been adopted for monitoring in the ICU, predominantly because of motor artifacts in non-paralyzed patients, and the lack of data demonstrating improved outcomes when bispectral index technology is used over standard sedation scales. Bispectral index monitors have found a unique niche in patients requiring sedation with concurrent neuromuscular blockers, in whom other validated sedation scales would not apply.

\section{Monitoring cognition}

Because of increased awareness of the neurocognitive complications of critical illness, evaluating and maintaining cognition in the ICU has also received considerable 
recent attention. Cognitive dysfunction can occur as a result of primary neurological disorders (e.g., stroke, head injury), or secondary neurologic complications resulting from critical illness (e.g., delirium, encephalopathy, hypoxia). Delirium, perhaps the best studied etiology of ICU-related neurocognitive dysfunction, is defined by the Diagnostic and Statistical Manual for Mental Disorders as the following: A disturbance of consciousness with reduced ability to focus, sustain or shift attention; a change in cognition or the development of a perceptual disturbance; a disturbance with a rapid onset and fluctuating nature; evidence that the disruption is caused by either a general medical condition, intoxication or drug-withdrawal [27]. The Confusion Assessment Method for the ICU (CAM-ICU) is a validated tool developed to screen patients for delirium, including patients who are unable to communicate verbally because of intubation $[27,28]$. CAM-ICU uses the following to identify delirium: Acute onset or fluctuating mental status, inattention, disorganized thinking, and an altered level of consciousness $[27,28]$. Not only is CAM-ICU a validated assessment of delirium, but it has also been shown to be a predictor of outcomes in ventilated and non-ventilated patients $[29,30]$. Other validated delirium screening tools include the Intensive Care Delirium Screening Checklist (ICDSC) [31]. One potential advantage of the ICDSC compared to the CAM-ICU is that it permits a continuous or graded assessment of delirium (score 0 to 8 ), as opposed to a categorical assignment, thus offering the advantage of identifying patients with subsyndromal delirium, who are also at risk of poor neurocognitive outcomes. Although studies have shown that delirium is a predictor of poor outcome, it remains unknown whether prevention or appropriate treatment will result in improved outcomes, and this important issue is worthy of further research. Whereas validated tools exist to detect the presence or absence of delirium in the ICU (one potential cause of cognitive dysfunction), limited tools are available at present outside of a formal neurological exam to monitor or quantify cognition itself in the critically ill.

One standard tool that offers a rapid assessment of cognition over a period of 5 to 10 minutes is the Mini Mental State Exam (MMSE), which divides cognition into 5 domains: Orientation, registration, attention and calculation, recall and language [32]. This scale was developed primarily as a screening tool for the presence of dementia in outpatients, and application of this instrument in the ICU is difficult given the requirement for verbal responses. The Adapted Cognitive Exam (ACE) is a scale that has recently been developed and validated to evaluate cognition in the critically ill [33]. It was modeled on the MMSE using the same five domains, but tailored to critically ill patients in such a way that it could be performed in patients who were non-verbal. Like the MMSE, it evaluates cognition on a numerical scale. The ACE has many potential clinical applications, and is an important measurement tool that can be applied to help better understand the continuum of neurocognitive sequelae starting at entry to the ICU through recovery and long-term follow up. This scale was utilized in the ANIST study to assess the differential effects on cognition of two different sedatives (dexmedetomidine and propofol) administered to achieve the same level of sedation [15].

\section{Sedative agents}

Many agents have been used for sedation, analgesia, and anxiolysis in the ICU. The most frequently used agents include members of the following drug or drug classes: Opioid, benzodiazepines, propofol and dexmedetomidine. When choosing an agent for ICU sedation, multiple factors must be taken into consideration. Typical considerations include indication for sedation, onset of action, duration of action, route of elimination, drug interactions, and adverse effects. We submit that another (perhaps less commonly considered) criteria be considered: The potential impact of the sedative on cognition and neurological sequelae, and the likelihood of the agent (appropriately dosed) to achieve a state of 'cooperative sedation. A comprehensive review of the pharmacokinetics and dynamics of these medications is beyond the scope of this review; however, details for individual agents can be found in Table 1 [34].

\section{Comparative trials}

Despite the many options for sedative agents, no one agent has been identified as superior in all cases. There are, however, several important trials comparing agents. The Maximizing Efficacy of Targeted Sedation and Reducing Neurological Dysfunction (MENDS) trial was the first randomized, double-blind trial evaluating the effect of sedative agents on delirium-free and coma-free days [35]. The two agents compared in this trail were lorazepam and dexmedetomidine. For the primary outcome, use of dexmedetomidine was associated with a greater number of delirium-free and coma-free days; however, this difference was driven by the number of coma-free days, as the number of delirium-free days was similar between groups. No differences in outcomes, including duration of mechanical ventilation, ICU LOS, or 28-day mortality were seen. One major limitation of the trial was the depth of sedation, which was chosen by the primary team. Baseline RASS scores for the dexmedetomidine and lorazepam groups were -3 and -4 respectively [35]. Had lighter or 'cooperative' sedation goals been achieved (closer to 0 to -1 ), the incidence of drug-induced coma would likely have been less (particularly with lorazepam), and results may have 
Table 1. Key characteristics of agents commonly used for sedation in intensive care unit (ICU) patients

\begin{tabular}{|c|c|c|c|c|c|c|}
\hline Drug & $\begin{array}{l}\text { Type of } \\
\text { medication }\end{array}$ & Sedation & Analgesia & Mechanism of action & Advantages & Adverse effects \\
\hline Fentanyl & Opioid & + & +++ & Mu receptor agonist & $\begin{array}{l}\text { Reversible, rapid onset, } \\
\text { short duration }\end{array}$ & $\begin{array}{l}\text { Respiratory depression, } \\
\text { chest wall rigidity, gastric } \\
\text { dysmotility, hypotension }\end{array}$ \\
\hline Remifentanil & Opioid & + & +++ & Mu receptor agonist & $\begin{array}{l}\text { Reversible, rapid onset, } \\
\text { short duration }\end{array}$ & $\begin{array}{l}\text { Respiratory depression, } \\
\text { chest wall rigidity, gastric } \\
\text { dysmotility, hypotension }\end{array}$ \\
\hline Morphine sulfate & Opioid & + & +++ & Mu receptor agonist & Reversible & $\begin{array}{l}\text { Respiratory depression, } \\
\text { gastric dysmotility, } \\
\text { hypotension, hallucinations }\end{array}$ \\
\hline Diazepam & Benzodiazepine & +++ & + & GABA $A_{a}$ receptor agonist & Reversible & $\begin{array}{l}\text { Respiratory depression, } \\
\text { hypotension, confusion }\end{array}$ \\
\hline Lorazepam & Benzodiazepine & +++ & - & GABA r receptor agonist & Reversible & $\begin{array}{l}\text { Respiratory depression, } \\
\text { hypotension, confusion }\end{array}$ \\
\hline Midazolam & Benzodiazepine & +++ & - & $\mathrm{GABA}_{\mathrm{a}}$ receptor agonist & $\begin{array}{l}\text { Reversible, shorter duration, } \\
\text { and titratable }\end{array}$ & $\begin{array}{l}\text { Respiratory depression, } \\
\text { hypotension, confusion }\end{array}$ \\
\hline Dexmedetomidine & Alpha-2 agonist & ++ & ++ & $\begin{array}{l}\text { Alpha-2 receptor agonist } \\
\text { (pre- and post-synaptic }\end{array}$ & Maintains cognitive function & $\begin{array}{l}\text { Dry mouth, bradycardia, } \\
\text { hypotension, adrenal } \\
\text { suppression, atrial } \\
\text { fibrillation }\end{array}$ \\
\hline Propofol & & +++ & - & Unclear & $\begin{array}{l}\text { Very short duration, easy } \\
\text { to titrate }\end{array}$ & $\begin{array}{l}\text { Hypotension, respiratory } \\
\text { depression, metabolic } \\
\text { acidosis, rhabdomyolysis, } \\
\text { anaphylaxis, sepsis, pain at } \\
\text { injection site }\end{array}$ \\
\hline
\end{tabular}

differed. Another recent randomized, double-blind trial, the Safety and Efficacy of Dexmedetomidine Compared with Midazolam (SEDCOM) trial, compared midazolam to dexmedetomidine [36], requiring daily awakening trials and a targeted RASS score of -2 to +1 . The primary outcome was percent time within RASS goal and secondary outcomes included assessment of delirium, duration of mechanical ventilation and ICU LOS. Whereas there was no difference in the primary outcome, patients in the dexmedetomidine group had a lower prevalence of delirium and a larger number of delirium-free days. In addition, patients sedated with dexmedetomidine had a shorter time to extubation [36].

A final study that further highlights the additional cognitive benefits of dexmedetomidine was conducted as a double blind, cross-over trial that compared dexmedetomidine to propofol [15]. The primary endpoint of the ANIST trial was the change in cognition (as measured by the 100-point ACE test) with dexmedetomidine as compared to propofol. Patients were randomized to either dexmedetomidine or propofol to start, and after an appropriate washout period, switched to the other agent. Cognitive assessments were performed at baseline, after the washout phase, and on each drug (RASS $=0$ to -1 ) [15]. Sedation with propofol diminished ACE scores on average by 12.4 points, whereas dexmedetomidine improved patient cognition by 6.8 points (difference in cognitive score $=19.2[95 \%$ CI 12.3-26.1, $\mathrm{p}<0.001]$ ).
These results are important, and illustrate the differential impact of two different sedative agents (titrated to the same level of sedation) on cognition.

In general, benzodiazepines are thought to be the most closely associated with delirium, as supported by the SEDCOM trial [36], although other data have linked benzodiazepines and opioids to the onset and increased duration of delirium [37]. Although propofol is not associated with delirium, it does affect cognition as shown in the ANIST study [15]. Dexmedetomidine is superior to the alternatives when preservation of cognition and avoidance of delirium is necessary. Low dose opioid infusions may also be able to achieve this in certain patients.

\section{Practical considerations}

Despite recommendations for daily interruption of sedation and protocol-driven sedation, survey data suggest that clinical practice lags behind the evidence. The use of daily interruption for patients receiving continuous infusions occurs inconsistently, with surveys reporting 40 to $62 \%$ rates of compliance [38-41]. In addition, despite the perception that sedation scales are beneficial, only $47-60 \%$ of ICUs had implemented sedation protocols at the time of the survey [39-41], and $85.6 \%$ of physicians in a Brazilian survey indeed believed their patients were usually over-sedated [41]. The same survey reported sedation levels being assessed 2 times or less per day in over $50 \%$ of patients. 
Nursing support of sedation strategies is crucial since they are the providers implementing the sedation plan. According to a survey, most critical care nurses believe that sedation is necessary when patients are intubated, and some reported feeling influenced by family members of patients as it relates to sedation practices [42]. The impact of staffing and the pressure of performing other nursing functions were identified as influences for onethird of nurses [42]. Nurses are at the patient end of a caring and compassionate group of health care providers, so the reason behind light sedation, and the likely greater nursing effort involved, must be appropriately communicated at the time the plan is made.

Although light or 'cooperative' sedation necessitates an increased level of vigilance, the heightened awareness and intellectual capacity promotes patient autonomy. Despite the potential need for restraints in some circumstances, reduction in sedation may permit patients to be more involved in decision-making as it relates to their own healthcare. Some patients in fact may be appropriate candidates for the no-sedation strategies that showed improved outcome in one study [6]; but it is important to evaluate the safety measures of that group before implementing such a protocol. For example, Strom and colleagues [6] implemented the protocol of no-sedation in a unit where all patients had 1:1 nursing care, plus an additional person in the ICU to 'calm patients'. Some units may have to increase staffing in order to accommodate this practice; however, it may yet be a cost effective strategy considering the improved outcomes observed. Another intervention, early mobilization, requires a multidisciplinary team to execute safely and effectively, yet some investigators have found that implementing strategies for early mobilization is feasible without increase ICU staffing [11].

As far as which sedatives to consider, dexmedetomidine has been shown to be beneficial for general cognition, as measured by the ACE, and for minimizing delirium, although more costly than other sedative agents. As with any agent, careful consideration of a drug usage policy must consider cost-effectiveness, ensuring optimal use of the drug to maximize drug-related outcomes.

\section{Conclusion}

Despite data suggesting significant improvements with daily awakening trials and early mobilization, these practices are far from being universally adopted in critical care units. Implementation of these interventions into standard practice in individual ICUs takes the effort of a multidisciplinary team and in some cases increases expenditures for institutions. However, with the potential improvement in outcomes, these interventions have the potential to be cost-effective and have a positive impact on patient outcomes above and beyond ICU survival. The neurocognitive effects of critical illness continue to gain recognition and represent an area ripe for continued research. Existing data suggest that the choice of sedative and sedation strategy can have an impact on cognition, duration of ventilation, and outcomes in acute illness. Further research is needed to identify whether active strategies to minimize or treat cognitive abnormalities during the ICU stay will translate to better neurocognitive outcomes in the long term.

\section{Abbreviations}

ACE, Adapted Cognitive Exam; ANIST, Acute Neurological ICU Sedation Trial; ATICE, Adaptation to the Intensive Care Environment; ARDS, acute respiratory distress syndrome; $C A B G$, coronary artery bypass graft; $C A M-I C U$, Confusion Assessment Method for the ICU; EEG, electroencephalograph; ICDSC, Intensive Care Delerium Screening Checklist; ICP, intercranial pressure; ICU, intensive care unit; LOS, length of stay; MENDS, Maximizing Efficacy of Targeted Sedation and Reducing Neurological Dysfunction (MENDS); MMSE, Mini Mental State Exam; NICS, Nursing Instrument for the Communication of Sedation; PTSD, post-traumatic stress disorder; RASS, Richmond AgitationSedation Scale; SAS, Riker Sedation-Agitation Scale; SEDCOM, Safety and Efficacy of Dexmedetomidine Compared with Midazolam; SBT, spontaneous breathing trial.

\section{Competing interests}

The authors declare that they have no competing interests.

\section{Published: 20 March 2012}

\section{References}

1. Hopkins RO, Brett S: Chronic neurocognitive effects of critical illness. Curr Opin Crit Care 2005, 11:369-375.

2. Newman MF, Kirchner JL, Phillips-Bute B, et al (2001) Longitudinal assessment of neurocognitive function after coronary-artery bypass surgery. N Engl J Med 2001, 344: 395-402.

3. Hopkins RO, Weaver LK, Pope D, Orme JF, Bigler ED, Larson-Lohr V: Neuropsychological sequelae and impaired health status in survivors of severe acute respiratory distress syndrome. Am J Respir Crit Care Med 1999, 160:50-56.

4. Kress JP, Pohlman AS, O'Connor MF, Hall JB: Daily interruption of sedative infusions in critically ill patients undergoing mechanical ventilation. N Engl J Med 2000, 342:1471-1477

5. Girard TD, Kress JP, Fuchs BD, et al: Efficacy and safety of a paired sedation and ventilator weaning protocol for mechanically ventilated patients in intensive care (Awakening and Breathing Controlled trial): a randomized controlled trial. Lancet 2008, 371:126-134.

6. Strom T, Martinussen T, Toft P: A protocol of no sedation for critically ill patients receiving mechanical ventilation: a randomized trial. Lancet 2010, 375:475-480.

7. Jackson JC, Hart RP, Gordon SM, Hopkins RO, Girard TD, Ely EW: Posttraumatic stress disorder and post-traumatic stress symptoms following critical illness in medical intensive care unit patients: assessing the magnitude of the problem. Crit Care 2007, 11:R27.

8. Kress JP, Gehlback B, Lacy M, Pliskin N, Pohlman AS, Hall JB: The long-term psychological effects of daily sedative interruption on critically ill patients. Am J Respir Crit Care Med 2003, 168:1457-1461.

9. Rochester CL: Rehabilitation in the intensive care unit. Semin Respir Crit Care Med 2009, 30:656-669.

10. Herridge MS, Cheung AM, Tansey CM, et al: One year outcomes in survivors of the acute respiratory distress syndrome. N Engl J Med 2003, 348:683-693.

11. Bailey $P$, Thomsen GE, Spuhler VJ, et al: Early activity is feasible and safe in respiratory failure patients. Crit Care Med 2007, 35:139-145.

12. Thomsen GE, Snow GL, Rodriguez L, Hopkins RO: Patients with respiratory failure increase ambulation after transfer to an intensive care unit where early activity is a priority. Crit Care Med 2008, 36:1119-1124.

13. Morris PE, Goad A, Thompson C, et al: Early intensive care unit mobility therapy in the treatment of acute respiratory failure. Crit Care Med 2008, $36: 2238-2243$ 
14. Schweikert WD, Pohlman MC, Pohlman AS, et al: Early physical and occupational therapy in mechanically ventilated, critically ill patients: a randomized controlled trial. Lancet 2009, 373:1874-1882.

15. Mirski MA, Lewin JJ, LeDroux S, et al: Cognitive improvement during continuous sedation in critically ill, awake and responsive patients: the Acute Neurological ICU Sedation Trial (ANIST). Intensive Care Med 2010, 36:1505-1513.

16. Sessler CN, Gosnell MS, Grap MJ, et al: The Richmond Agitation-Sedation Scale: validity and reliability in adult intensive care unit patients. Am J Respir Crit Care Med 2002, 166:1338-1344.

17. Ely EW, Truman B, Shintani A, et al: Monitoring sedation status over time in ICU patients: reliability and validity of the Richmond Agitation-Sedation Scale (RASS). JAMA 2003, 289:2983-2991

18. Riker RR, Picard JT, Fraser GL: Prospective evaluation of the SedationAgitation Scale for the adult critically ill patients. Crit Care Med 1999, 27:1325-1329.

19. Devlin JW, Boleski G, Mlynarek M, et al: Motor Activity Assessment Scale: avalid and reliable sedation scale for use with mechanically ventilated patients in an adult surgical intensive care unit. Crit Care Med 1999, 27:1271-1275.

20. De Jonghe B, Cook D, Griffith L, et al: Adaptation to the intensive care environment (ATICE): development and validation of a new sedation assessment instrument. Crit Care Med 2003, 31:2344-2354.

21. Mirski MA, LeDroux SN, Lewin JJ, Thompson CB, Mirski KT, Griswold M: Validity and reliability of an intuitive conscious sedation scoring tool: The nursing instrument for the communication of sedation. Crit Care Med 2010, 38:1674-1684.

22. Myles PS, Leslie K, McNeil J, et al: Bispectral index monitoring to prevent awareness during anaesthesia: the B-Aware randomized controlled trial. Lancet 2004, 363:1757-1763.

23. Deogaonkar A, Gupta R, DeGorgia M, et al: Bispectral index monitoring correlates with sedation scales in brain-injured patients. Crit Care Med 2004, 32:2403-2406.

24. Olson DM, Thoyre SM, Peterson ED, Graffagnino C: A randomized evaluation of bispectral index-augmented sedation assessment in neurological patients. Neurocrit Care 2009, 11:20-27.

25. Riker RR, Fraser GL, Simmons LE, Wilkins ML: Validating the sedationagitation scale with the bispectral index and visual analog scale in adult ICU patients after cardiac surgery. Intensive Care Med 2001, 27:853-858.

26. Karachandani K, Rewari V, Trikha A, Batra RK: Bispectral index correlates well with Richmond agitation sedation scale in mechanically ventilated critically ill patients. J Anesth 2010, 24:394-398.

27. Ely EW, Margolin R, Francis J, et al: Evaluation of delirium in critically ill patients: validation of the confusion assessment method for the intensive care unit (CAM-ICU). Crit Care Med 2001, 29:1370-1379.

28. Ely EW, Inouye SK, Bernard GR, et al: Delirium in mechanically ventilated patients: validity and reliability of the confusion assessment method for the intensive care unit (CAM-ICU). JAMA 2001, 286:2703-2710.

29. Ely WE, Shintani A, Truman B, et al: Delirium as a predictor of mortality in mechanically ventilated patients in the intensive care unit. JAMA 2004, 291:1753-1762.
30. Thomason JWW, Shintani A, Peterson JF, Pun BT, Jackson JC, Ely WE: Intensive care unit delirium is an independent predictor of longer hospital stay: a prospective analysis of 261 non-ventilated patients. Crit Care 2005 9:R375-R381.

31. Ouimet S, Riker R, Bergeron N, Cossette M, Kavanagu B, Skrobik Y: Subsyndromal delirium in the ICU: evidence for a disease spectrum Intensive Care Med 2007, 33:1007-1013.

32. Folstein MF, Folstein SE, McHugh PR: Mini-Mental State: a practical method for grading the cognitive state of patients for the clinician. J Psychiat Res 1975, 12:189-198.

33. Lewin JJ, LeDroux SN, Shermock KM, et al: Validity and reliability of The Johns Hopkins Adapted Cognitive Exam for critically ill patients. Crit Care Med 2012, 40:139-144.

34. Mirski MA, Lewin JJ: Sedation and analgesia for the critically ill patient Contemp Crit Care 2007, 4:1-12.

35. Pandharipande PP, Pun BT, Herr DL, et al: Effect of sedation with dexemedetomidine vs lorazepam on acute brain dysfunction in mechanically ventilated patients: the MENDS randomized controlled trial JAMA 2007, 298:2644-2653

36. Riker RR, Shehabi Y, Bokesch PM, et al: Dexmedetomidine vs midazolam for sedation of critically ill patients. JAMA 2009, 301:489-499.

37. Pisani MA, Murphy TE, Araujo KL, Slattum P, Van Ness PH, Inouye SK: Benzodiazepine and opioid use and the duration of intensive care unit delirium in an older population. Crit Care Med 2009, 37:177-183.

38. Mehta S, Burry L, Fischer S, et al: Canadian survey of the use of sedatives, analgesics, and neuromuscular blocking agents in critically ill patients. Crit Care Med 2006, 34:374-380.

39. O'Connor M, Bucknall T, Manias E: Sedation management in Australian and New Zealand intensive care units: doctors' and nurses' practices and opinions. Am J Crit Care 2010, 19:285-295.

40. Salluh JIF, Dal-Pizzol F, Mello PVC, et al: Delirium recognition and sedation practices in critically ill patients: a survey on the attitudes of 1015 Brazilian critical care physicians. J Crit Care 2009, 24:556-562.

41. Tanios MA, de Wit M, Epstein SK, Devlin JW: Preceived barriers to the use of sedation protocols and daily interruptions: a multidisciplinary survey. J Crit Care 2009, 24:66-73.

42. Guttormson JL, Chlan L, Weinert C, Savik K: Factors influencing nurse sedation practices with mechanically ventilated patients: a US national survey. Intensive Crit Care Nurs 2010, 26:44-50.

doi:10.1186/cc11231

Cite this article as: Goodwin $\mathrm{H}$, et al.: 'Cooperative sedation': optimizing comfort while maximizing systemic and neurological function. Critical Care 2012, 16:217. 